\title{
Ultracongruent insert design is a safe alternative to posterior cruciate-substituting total knee arthroplasty: 5-year results of a randomized controlled trial
}

\author{
Jörg Lützner ${ }^{1}\left[\right.$ [ Franziska Beyer $^{1} \cdot$ Cornelia Lützner $^{1} \cdot$ Roman Riedel $^{1} \cdot$ Eric Tille $^{1}$
}

Received: 21 January 2021 / Accepted: 18 March 2021 / Published online: 11 April 2021

(c) The Author(s) 2021

\begin{abstract}
Purpose If substitution of the posterior cruciate ligament in total knee arthroplasty is necessary, there are two options available: posterior stabilized (PS) design with a post-cam mechanism or anterior-lipped ultracongruent (UC) inserts. UC inserts have the advantage that no femoral box is necessary and a standard femoral component can be used. The aim of this study was to compare the range of motion (ROM) and patient-reported outcome (PRO) after UC and PS fixed-bearing TKA. Better ROM in PS TKA and no difference in PRO between both designs was hypothesized.

Methods A randomized controlled trial with 127 patients receiving a fixed-bearing UC or PS design of the same knee system was performed. Nine patients died and there were four revision surgeries. 107 patients completed the 5-year follow-up. Patient-reported outcome was assessed. Patellofemoral problems were evaluated using selected applicable questions of the Oxford Knee Score (getting up from a table, kneeling, climbing stairs).

Results Surgical time was 10 min shorter in the UC group $(p<0.001)$. After 5 years, both groups demonstrated good knee function and health-related quality of life without significant differences between the groups. Both groups demonstrated a high satisfaction score and the majority of patients would undergo this surgery again. Patellofemoral problems were recognized more frequently in the PS group $(p=0.025)$.

Conclusion Both designs demonstrated similar good results after 5 years. Stabilization with an anterior-lipped UC insert can be considered a safe alternative to the well-established PS design if cruciate substitution is necessary.

Level of evidence 1 .
\end{abstract}

Keywords TKA · TKR · Anterior stabilized · Posterior stabilized · Ultracongruent insert · Deep dished insert · Stability · Range of motion $\cdot$ Results $\cdot$ Patient-reported outcome

Jörg Lützner

Joerg.Luetzner@ukdd.de

Franziska Beyer

Franziska.Beyer@ukdd.de

Cornelia Lützner

Cornelia.Luetzner@ukdd.de

Roman Riedel

Roman.Riedel@ukdd.de

Eric Tille

Eric.Tille@ukdd.de

1 University Center of Orthopaedic, Trauma and Plastic

Surgery, University Hospital Carl Gustav Carus, TU

Dresden, Fetscherstr. 74, 01307 Dresden, Germany

\section{Introduction}

The decision to sacrifice or preserve the posterior cruciate ligament (PCL) in total knee arthroplasty (TKA) mainly depends on surgeon preference. Despite better kinematic performance of PS TKA, no difference in outcome has been demonstrated between retention and sacrifice of the PCL except for slightly better knee flexion in PS TKA [6]. However, if the PCL is insufficient, absent, or needs to be resected, the substitution of the PCL is necessary $[4,20$, 26]. In these situations, there are two options for substitution of the PCL: posterior-stabilized (PS) implants with a box and cam mechanism or anterior-lipped ultracongruent (UC) inserts. The PS design needs additional bone resection of the femur for preparation of the box which increases surgical time and fracture risk [20]. Furthermore, there is additional 
cam-mechanism polyethylene wear, a risk of dislocation [22] and the patella clunk syndrome may occur [16]. The use of a highly conforming ultracongruent (UC) insert with a standard femoral component is a simple alternative to the established PS TKA design $[15,20]$. However, the UC design has potential disadvantages. While some studies demonstrated a reduced range of motion (ROM) for UC compared to PS design [24, 32], other studies found similar ROM for both designs $[18,19]$. Some studies found reduced axial rotation in UC compared to PS TKA [11, 18, 25], which might result in increased long-term polyethylene (PE) wear in a highly congruent fixed-bearing design. For many TKA brands, anterior-lipped UC inserts are available. Despite the regular use of these UC inserts, there is only limited evidence about mid- or long-term outcome. Only few studies report 5- or 10 -year results, none with an appropriate control group [3, $10,18]$.

\section{Purpose and hypothesis}

The aim of this study was to compare the range of motion (ROM) and patient-reported outcome (PRO) after UC and PS fixed-bearing TKA. Better ROM in PS TKA and no difference in PRO between both designs was hypothesized.

\section{Materials and methods}

In a randomized controlled trial [23], all patients scheduled for a condylar TKA were screened. Patients with the need for a higher constraint TKA (condylar constraint or rotating hinge TKA) were not included. A total of 127 patients were included. Patients were interviewed by a trained study nurse before surgery, 3 months, as well as 1, 3 and 5 years after surgery. Nine patients died, four patients required revision surgery and seven patients were lost to follow-up, resulting in 51 patients with an UC and 56 patients with a PS design at the final follow-up (Fig. 1). ROM was measured with a goniometer preoperatively and at each follow-up. Knee Function (Oxford Knee Score, OKS [28]), physical activity (University of Los Angeles (UCLA) activity scale [2]) and health-related quality of life (SF36 [9]) were obtained. At the final follow-up, patients were asked how satisfied they were with the outcome of TKA. It was assessed on a visual analogue scale from 0 (very dissatisfied) to 10 (very satisfied) [8]. Additionally, patients were asked if they would undergo TKA again, if necessary. Patients could choose between five possible answers: definitely yes, possibly yes, not sure, probably not, certainly not [13]. Sociodemographic data, operative details and revisions were assessed for all patients. Surgical time was measured from skin incision to wound closure.
The surgeries were performed by two experienced arthroplasty surgeons. All patients received a cemented fixedbearing TKA (Columbus, B. Braun Aesculap, Tuttlingen, Germany) without patellar resurfacing. Both cruciate ligaments were resected. A gap-balancing technique was used with the help of a navigation system (Orthopilot, Software TKR v4.3, B. Braun Aesculap, Tuttlingen, Germany). Tibial and femoral bone cuts, sizing of the femoral component and soft-tissue balancing were performed as indicated by the navigation system. For the UC design a standard femoral component was used, for the PS design additional preparation of the box was performed.

The study was registered at ClinicalTrials.gov (NCT04679857). All patients signed an informed consent.

\section{Statistical analysis}

The sample size was calculated to detect a minimum clinically relevant difference of $5^{\circ}$ of knee flexion between the two implant designs. With a power of $80 \%$, a significance level of 0.05 and an estimated standard deviation of $10^{\circ}$, a number of 64 patients in each group were necessary.

Patient characteristics, data from TKA surgery, comorbidities (ASA grade) and adverse events were recorded prospectively, assembled into a database together with the questionnaires and analysed using SPSS ${ }^{\circledR}$ software (release 26 for Windows ${ }^{\circledR}$ ). The acquired data did not show a normal distribution (Kolmogorov-Smirnov test) and therefore reported as median, 25th and 75th percentiles for continuous values and absolute and relative frequencies for categorical values, respectively. To compare study groups, Mann-Whitney $U$ test for continuous values and chi-square test for categorical values were used.

\section{Results}

There were no differences between the study groups 5 years postoperatively regarding age, gender, BMI and comorbidities. Surgical time was 10 min longer in the PS group $(p<0.001$, Table 1).

Knee flexion increased from a median $105^{\circ}$ in the UC group and $100^{\circ}$ in the PS group before surgery to $115^{\circ}$ in both groups at the final follow-up. Knee function (OKS), activity (UCLA activity scale) and health-related quality of life (SF36) demonstrated significant improvement in both groups without significant differences (Table 2).

The satisfaction score was high in both groups. The majority of patients $(89.7 \%)$ indicated that they would undergo TKA surgery again, if necessary (Fig. 2).

Looking at the items in the OKS regarding activities with a high patellofemoral load, there were better results for the UC design for getting up from a table $(p=0.025)$. Patients in 
Fig. 1 Flow chart of study patients

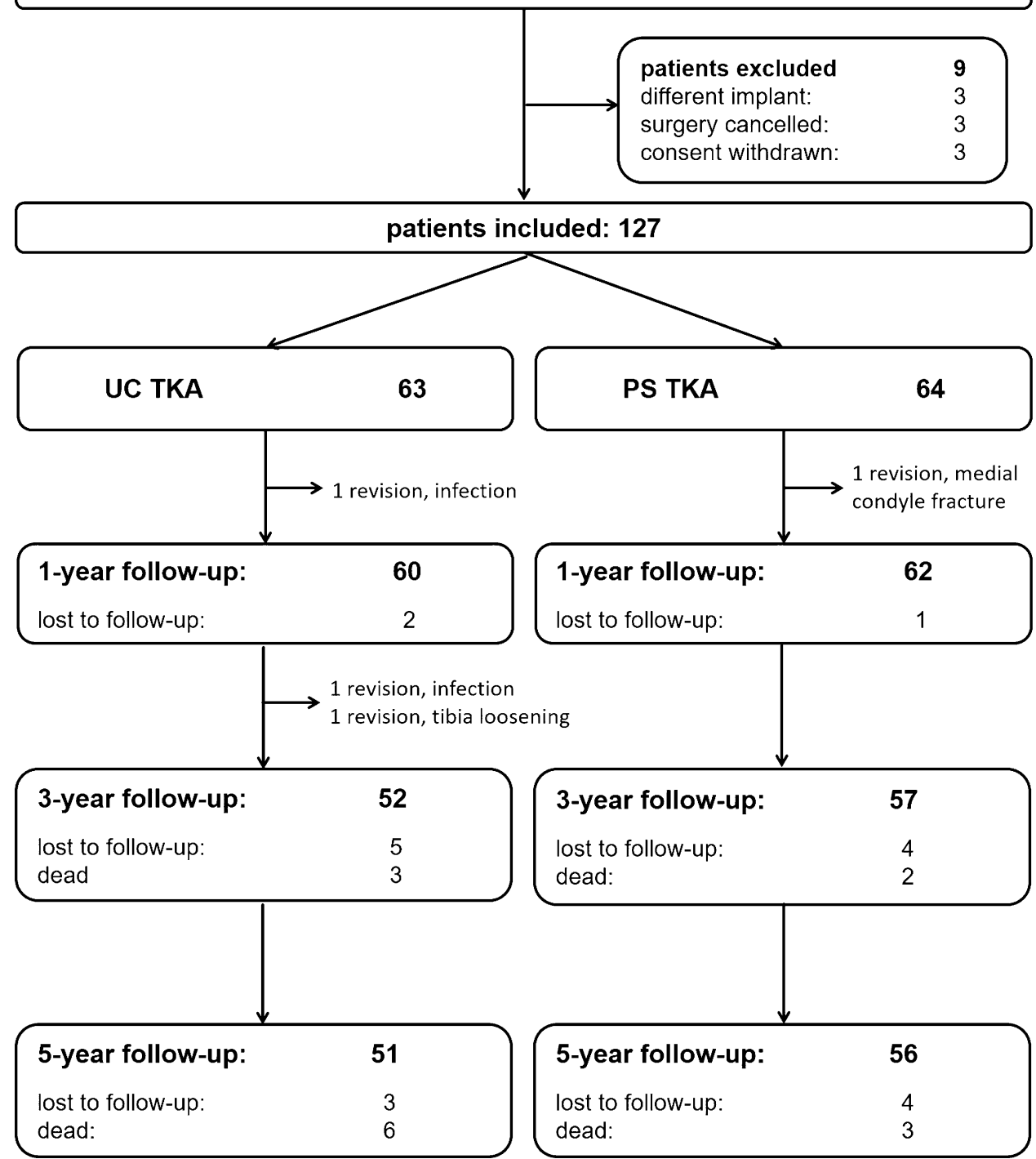

Table 1 Patient characteristics, given as median (25th percentile, 75th percentile) and relative frequencies

\begin{tabular}{llll}
\hline Patient characteristics & UC TKA & PS TKA & $p$ value \\
\hline Age at surgery (years) & $71(62 ; 77)$ & $72(62 ; 77)$ & n.s \\
Gender (female) & $72.5 \%$ & $64.3 \%$ & n.s \\
BMI $\left(\mathrm{kg} / \mathrm{m}^{2}\right)$ & $31.1(27.9 ; 35.4)$ & $29.4(27.6 ; 34.1)$ & $\mathrm{n} . \mathrm{s}$ \\
ASA grade & & & \\
1 and 2 & $47.1 \%$ & $44.6 \%$ & n.s \\
3 and 4 & $52.9 \%$ & $55.4 \%$ & \\
Surgical time (min) & $86(80 ; 94)$ & $96(88 ; 101)$ & $<0.001$ \\
\hline
\end{tabular}

both groups had the greatest difficulties in kneeling, which was impossible or extremely difficult in $51 \%$ in the UC group and $70 \%$ in the PS group ( $p=0.052$, Fig. 3$)$.
Four patients required revision surgery until the 5-year follow-up. One patient in the PS group experienced a medial condyle fracture 3 months after surgery, which might have been missed during the initial surgery. Two patients in the UC group had a deep infection and staged revision TKA was performed. One patient in the UC group required partial revision of the tibial component due to aseptic loosening 29 months after surgery.

\section{Discussion}

The most important finding of this study was that there was no difference in ROM, knee function, physical activity, health-related QoL or satisfaction in fixed-bearing TKA after 5 years using either UC or PS design. 
Table 2 PRO before surgery, and 1, 3 and 5 years after surgery, given as median values ( 25 th percentile, 75 th percentile)

\begin{tabular}{|c|c|c|c|}
\hline Score & UC TKA & PS TKA & $p$ value \\
\hline \multicolumn{4}{|c|}{ Oxford Knee Score (max. 48 points) } \\
\hline Before surgery & $20(17,25)$ & $22(18,25)$ & 0.370 \\
\hline 1-year follow-up & $42(36,45)$ & $37(29,43)$ & 0.004 \\
\hline 3-year follow-up & $42(36,46)$ & $38(33,42)$ & 0.012 \\
\hline 5-year follow-up & $42(37,45)$ & $41(27,44)$ & 0.101 \\
\hline Improvement & $19(14,26)$ & $17(10,22)$ & 0.034 \\
\hline \multicolumn{4}{|c|}{ OKS pain component (max. 100 points) } \\
\hline Before surgery & $39(29,54)$ & $41(29,50)$ & 0.795 \\
\hline 1-year follow-up & $93(79,100)$ & $86(64,93)$ & 0.008 \\
\hline 3-year follow-up & $93(82,100)$ & $82(75,96)$ & 0.037 \\
\hline 5-year follow-up & $93(82,100)$ & $93(68,96)$ & 0.335 \\
\hline Improvement & $46(36,61)$ & $46(32,96)$ & 0.314 \\
\hline \multicolumn{4}{|c|}{ OKS function component (max. 100 points) } \\
\hline Before surgery & $45(35,55)$ & $50(38,60)$ & 0.123 \\
\hline 1-year follow-up & $80(65,90)$ & $70(55,83)$ & 0.009 \\
\hline 3-year follow-up & $80(65,95)$ & $70(60,80)$ & 0.049 \\
\hline 5 -year follow-up & $80(60,95)$ & $70(48,85)$ & 0.069 \\
\hline Improvement & $30(20,40)$ & $15(5,35)$ & 0.004 \\
\hline \multicolumn{4}{|c|}{ UCLA activity scale (max. 10 points) } \\
\hline Before surgery & $4(3,5)$ & $4(3,5)$ & 0.954 \\
\hline 1-year follow-up & $5(3,6)$ & $5(3,6)$ & 0.807 \\
\hline 3-year follow-up & $5(3,6)$ & $5(3,6)$ & 0.475 \\
\hline 5-year follow-up & $4(4,5)$ & $4(3,6)$ & 0.511 \\
\hline Improvement & $1(-1,2)$ & $1(0,2)$ & 0.639 \\
\hline \multicolumn{4}{|l|}{ SF36 physical scale ${ }^{a}$} \\
\hline Before surgery & $23.8(18.9,31.8)$ & $25.6(19.4,31.8)$ & 0.635 \\
\hline 1-year follow-up & $48(33.6,53.5)$ & $39.6(29,50.3)$ & 0.045 \\
\hline 3-year follow-up & $38.5(25.7,52.3)$ & $37.2(28.8,46)$ & 0.737 \\
\hline 5-year follow-up & $42.3(27.4,52.3)$ & $37.9(24.5,50.1)$ & 0.275 \\
\hline Improvement & $14.2(8.2,24.3)$ & $10.6(2,19.4)$ & 0.112 \\
\hline \multicolumn{4}{|l|}{ SF36 mental scale ${ }^{b}$} \\
\hline Before surgery & $57.8(46,65.8)$ & $56.4(46.9,63.9)$ & 0.585 \\
\hline 1-year follow-up & $55.7(43.9,59.6)$ & $53.2(43.7,58.1)$ & 0.450 \\
\hline 3-year follow-up & $54.7(43.7,59.4)$ & $53.4(46.8,59.9)$ & 0.684 \\
\hline 5-year follow-up & $56.5(49.5,62)$ & $54.5(48.4,60.3)$ & 0.388 \\
\hline Improvement & $-3.1(-9.7,6.3)$ & $-3.1(-8.4,5.2)$ & 0.820 \\
\hline \multicolumn{4}{|c|}{ Satisfaction with TKA (max. 10.0) } \\
\hline 5-year follow-up & $9.0(7.5,10.0)$ & $8.8(7.3,9.5)$ & 0.269 \\
\hline
\end{tabular}

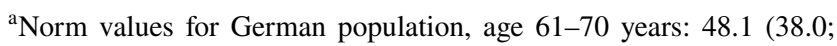
53.7), age > 70 years: $40.8(31.0 ; 50.1)$

${ }^{\mathrm{b}}$ Norm values for German population, age 61-70 years: 55.1 (50.0; $58.4)$, age $>70$ years: $53.6(48.9 ; 58.9)$

The use of cruciate retaining or substituting TKA is a matter of surgeon philosophy. Despite better reproduction of the femoral rollback during flexion and therefore more natural knee kinematics in PS TKA, no conclusive evidence supports the superiority of one design above the other in clinical practice [5, 27]. Regardless of surgeon preferences for one design, there are certain situations in which substitution of the PCL is obligatory: if the PCL is damaged, absent or needs to be resected. Bae et al. have analysed a series of 920 patients planned for a cruciate-retaining TKA [4]. In 83 knees (9\%) an intraoperative conversion to a PS design was necessary. In case of unplanned need for PCL substitution, the UC design offers advantages compared to the traditional PS design: no additional bone resection is necessary and a standard femoral component can be used making additional implant storage in the OR obsolete.

Kinematic studies demonstrated better ROM, less anteroposterior laxity and more posterior femoral rollback of PS compared to UC TKA $[5,11,17]$. These kinematic aspects seem to have no impact on clinical and patient-reported outcome in either approach of TKA. Kim et al. compared UC and PS TKA in the same patient and found no difference in side preference, satisfaction or joint perception despite kinematic advantages of the PS design [17]. Akti et al. investigated isokinetic performance and found no difference between UC and PS TKA [1]. Several studies compared short-time results between UC and PS TKA [1, 7, 17, 18, 20, 23, 24, 29, 30]. In these studies, different TKA designs were investigated and different patient-reported outcome measures were used, but all studies reported similar results for UC and PS TKA. There were no differences in revision rates or KOOS-JR scores between PS and UC TKA after mean 43 months follow-up in a large consecutive series of 5970 patients by Yacovelli et al. [33]. Only one study reported long-term results including fixed-bearing $(n=38)$ and mobile-bearing UC TKA $(n=199)$ [3]. There was no difference between all implant designs in survival after 10 years. Unfortunately, UC TKA is not clearly reported separately in the major arthroplasty registries. Looking at survivorship in the registries, there are generally higher revision rates for higher constraint TKA. In the Australian Arthroplasty Registry, high-volume surgeons preferring PS TKA had a $45 \%$ higher risk of revision at 13 years compared to surgeons preferring minimally stabilised TKA [31]. Two recent reviews $[5,27]$ summarized available evidence and found no difference in clinical outcome between UC and PS TKA which is consistent with the results of the present study. After reviewing kinematic and clinical studies as well as registry data, Meneghini et al. [27] concluded that a postcam mechanism is no longer necessary in modern primary TKA. These results suggest that UC TKA is a safe option.

While there was no overall difference between both groups, activities with high patellofemoral load (getting up, kneeling, climbing stairs) appeared to be more troublesome for patients in the PS group. The majority of patients in both groups had severe difficulties with or were unable to kneel. In vitro studies demonstrated that changes in patellofemoral kinematics and patellofemoral pressure increase after TKA depending on TKA design. This may have an influence on 
Fig. 2 Willingness to undergo the surgery again (if necessary)
Fig. 3 Questions from the OKS which are linked to patellofemoral problems
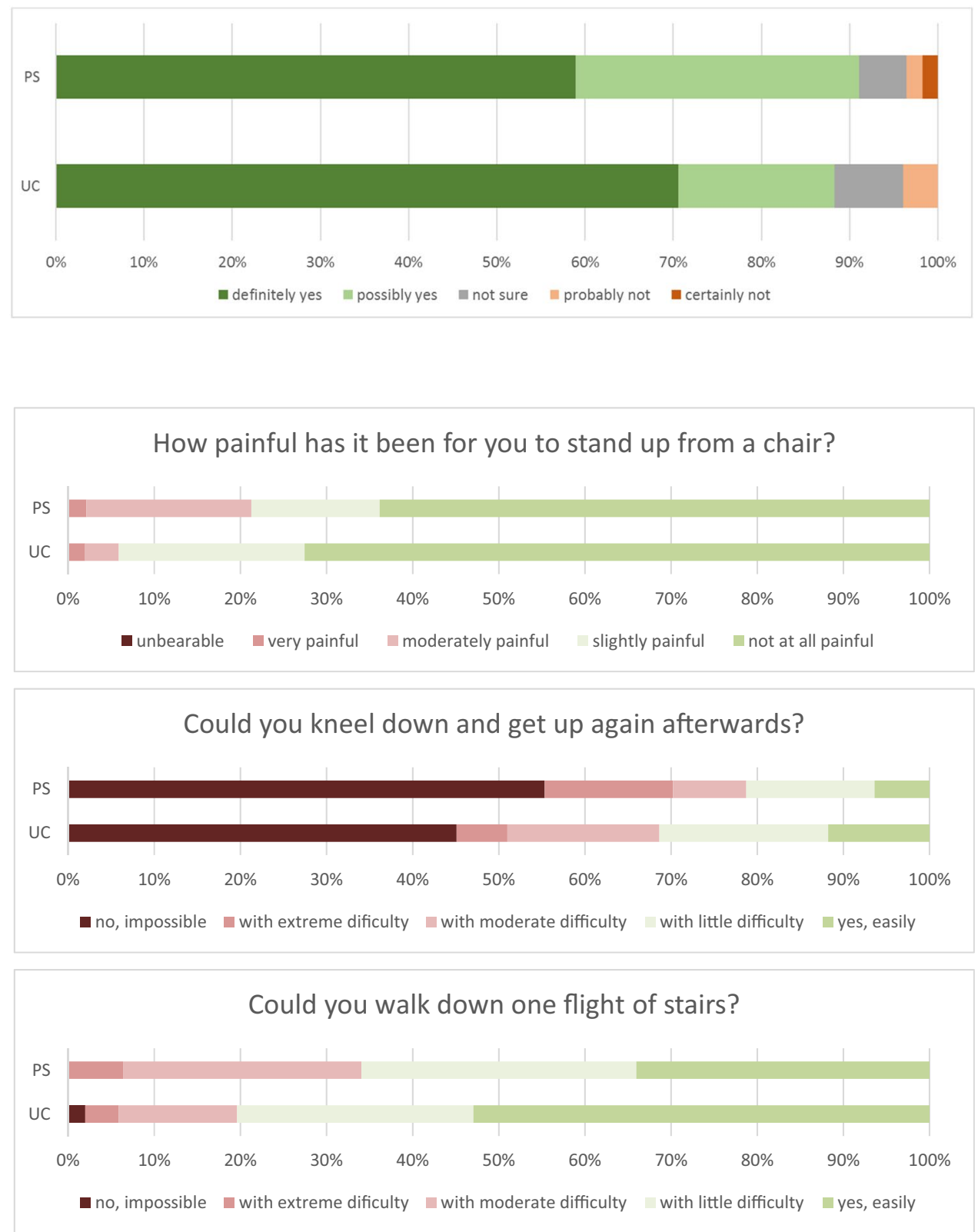

patellofemoral pain $[12,21]$. In vitro measurements demonstrated higher patellofemoral pressure in UC TKA compared with the PS design [14]. This is in contrast to the present study and might be explained by differences in the design of the anterior femur between implant brands and whether patellar resurfacing was performed or not. In the present study, no patellar resurfacing was performed. The patellar clunk syndrome has been described as a result of a large box design in PS TKA [16]. Consequently, the box design and the design of the anterior femur has been changed to become more "patella-friendly". Consequently, this does not seem to be an issue in modern PS design any longer.

This study has some limitations. Not all eligible patients could be included and few patients were lost to follow-up, therefore a risk of selection bias exists. This study was performed using a specific fixed-bearing TKA design. Results may therefore not be applicable to other TKA designs. Patellofemoral problems were assessed using the questions from the OKS. No specific validated score for patellofemoral pain was used. Despite these limitations, this study provides detailed information on mid-term outcome of UC and PS TKA. 


\section{Conclusion}

This study demonstrated no differences in ROM and PRO between fixed-bearing UC and PS TKA during mid-term follow-up. UC TKA can be considered a safe alternative to the well-established PS design in PCL substituting TKA. For surgeons who do not always substitute the PCL, this may be an advantage.

Acknowledgements The authors are grateful to B. Braun Aesculap for a research Grant and to Brit Brethfeld for her valuable assistance during follow-up and data management.

Funding Open Access funding provided by Projekt DEAL. This study was supported by a research grant from BBraun Aesculap.

\section{Declarations}

Conflict of interest JL has received research grants from Aesculap, Link, Mathys, Smith \& Nephew and Zimmer and honoraria from Aesculap, Link, Mathys and Pfizer.

Ethical approval The study has been performed in compliance with the Helsinki Declaration and has been approved by the local ethics committee (EK 227072012).

Open Access This article is licensed under a Creative Commons Attribution 4.0 International License, which permits use, sharing, adaptation, distribution and reproduction in any medium or format, as long as you give appropriate credit to the original author(s) and the source, provide a link to the Creative Commons licence, and indicate if changes were made. The images or other third party material in this article are included in the article's Creative Commons licence, unless indicated otherwise in a credit line to the material. If material is not included in the article's Creative Commons licence and your intended use is not permitted by statutory regulation or exceeds the permitted use, you will need to obtain permission directly from the copyright holder. To view a copy of this licence, visit http://creativecommons.org/licenses/by/4.0/.

\section{References}

1. Akti S, Karakus D, Sezgin EA, Cankaya D (2020) No differences in clinical outcomes or isokinetic performance between cruciate-substituting ultra-congruent and posterior stabilized total knee arthroplasties: a randomized controlled trial. Knee Surg Sports Traumatol Arthrosc. https://doi.org/10.1007/ s00167-020-06275-z

2. Amstutz HC, Thomas BJ, Jinnah R, Kim W, Grogan T, Yale C (1984) Treatment of primary osteoarthritis of the hip. A comparison of total joint and surface replacement arthroplasty. J Bone Jt Surg Am 66:228-241

3. Argenson J-N, Boisgard S, Parratte S, Descamps S, Bercovy M, Bonnevialle P et al (2013) Survival analysis of total knee arthroplasty at a minimum 10 years' follow-up: a multicenter French nationwide study including 846 cases. Orthop Traumatol Surg Res 99:385-390

4. Bae DK, Song SJ, Kim KI, Hur D, Lee HH (2016) Intraoperative factors affecting conversion from cruciate retaining to cruciate substituting in total knee arthroplasty. Knee Surg Sports Traumatol Arthrosc 24:3247-3253

5. Bae J-H, Yoon J-R, Sung J-H, Shin Y-S (2018) Posterior-stabilized inserts are preferable to cruciate-substituting ultracongruent inserts due to more favourable kinematics and stability. Knee Surg Sports Traumatol Arthrosc 26:3300-3310

6. Bercik MJ, Joshi A, Parvizi J (2013) Posterior cruciate-retaining versus posterior-stabilized total knee arthroplasty: a meta-analysis. J Arthroplasty 28:439-444

7. Biyani RK, Ziemba-Davis M, Ireland PH, Meneghini RM (2017) Does an anterior-lipped tibial insert adequately substitute for a post-cam articulation in total knee arthroplasty. Surg Tech Int 30:341-345

8. Bullens PH, van Loon CJ, de Waal Malefijt MC, Laan RF, Veth RP (2001) Patient satisfaction after total knee arthroplasty: a comparison between subjective and objective outcome assessments. J Arthroplasty 16:740-747

9. Bullinger M (1995) German translation and psychometric testing of the SF-36 Health Survey: preliminary results from the IQOLA Project. International Quality of Life Assessment. Soc Sci Med 41:1359-1366

10. Chavoix J-B (2013) Functionality and safety of an ultra-congruent rotating platform knee prosthesis at 5.6 years: more than 5 -year follow-up of the e. motion ${ }^{\circledR}$ UC-TKA. Open Orthop J 7:152

11. Fritzsche H, Beyer F, Postler A, Lützner J (2018) Different intraoperative kinematics, stability, and range of motion between cruciate-substituting ultracongruent and posterior-stabilized total knee arthroplasty. Knee Surg Sports Traumatol Arthrosc 26:1465-1470

12. Glogaza A, Schröder C, Woiczinski M, Müller P, Jansson V, Steinbrück A (2018) Medial stabilized and posterior stabilized TKA affect patellofemoral kinematics and retropatellar pressure distribution differently. Knee Surg Sports Traumatol Arthrosc 26:1743-1750

13. Hamilton D, Lane JV, Gaston P, Patton J, Macdonald D, Simpson A et al (2013) What determines patient satisfaction with surgery? A prospective cohort study of 4709 patients following total joint replacement. BMJ Open 3:e002525

14. Heyse TJ, Becher C, Kron N, Ostermeier S, Hurschler C, Schofer MD et al (2010) Patellofemoral pressure after TKA in vitro: highly conforming vs. posterior stabilized inlays. Arch Orthop Trauma Surg 130:191

15. Hofmann AA, Tkach TK, Evanich CJ, Camargo MP (2000) Posterior stabilization in total knee arthroplasty with use of an ultracongruent polyethylene insert. J Arthroplasty 15:576-583

16. Hozack W, Rothman RH, Booth JRRE, Balderston RA (1989) The patellar clunk syndrome: a complication of posterior stabilized total knee arthropiasty. Clin Orthop Relat Res 241:203-208

17. Kim MS, Koh IJ, Kim CK, Choi KY, Jeon JH, In Y (2020) Comparison of joint perception between posterior-stabilized and ultracongruent total knee arthroplasty in the same patient. J Bone Jt Surg 103:44-52

18. Kim TW, Lee SM, Seong SC, Lee S, Jang J, Lee MC (2016) Different intraoperative kinematics with comparable clinical outcomes of ultracongruent and posterior stabilized mobile-bearing total knee arthroplasty. Knee Surg Sports Traumatol Arthrosc 24:3036-3043

19. Ko Y-B, Jang E-C, Park S-M, Kim SH, Kwak Y-H, Lee H-J (2015) No difference in clinical and radiologic outcomes after total knee arthroplasty with a new ultra-congruent mobile bearing system and rotating platform mobile bearing systems after minimum 5-year follow-up. J Arthroplasty 30:379-383

20. Laskin RS, Maruyama Y, Villaneuva M, Bourne R (2000) Deepdish congruent tibial component use in total knee arthroplasty: a randomized prospective study. Clin Orthop Relat Res 380:36-44 
21. Leichtle UG, Wünschel M, Leichtle CI, Müller O, Kohler P, Wülker $\mathrm{N}$ et al (2014) Increased patellofemoral pressure after TKA: an in vitro study. Knee Surg Sports Traumatol Arthrosc 22:500-508

22. Lombardi AV Jr, Mallory TH, Vaughn BK, Krugel R, Honkala TK, Sorscher M et al (1993) Dislocation following primary posterior-stabilized total knee arthroplasty. J Arthroplasty 8:633-639

23. Lützner J, Beyer F, Dexel J, Fritzsche H, Lützner C, Kirschner S (2017) No difference in range of motion between ultracongruent and posterior stabilized design in total knee arthroplasty: a randomized controlled trial. Knee Surg Sports Traumatol Arthrosc 25:3515-3521

24. Machhindra MV, Kang JY, Kang YG, Chowdhry M, Kim TK (2015) Functional outcomes of a new mobile-bearing ultra-congruent TKA system: comparison with the posterior stabilized system. J Arthroplasty 30:2137-2142

25. Massin P, Boyer P, Sabourin M (2012) Less femorotibial rotation and AP translation in deep-dished total knee arthroplasty. An intraoperative kinematic study using navigation. Knee Surg Sports Traumatol Arthrosc 20:1714-1719

26. Matziolis G, Mehlhorn S, Schattat N, Diederichs G, Hube R, Perka C et al (2012) How much of the PCL is really preserved during the tibial cut? Knee Surg Sports Traumatol Arthrosc 20:1083-1086

27. Meneghini RM, Stefl MD, Hodge WA, Banks SA (2019) A campost mechanism is no longer necessary in modern primary total knee arthroplasty. J Knee Surg 32:710-713

28. Naal FD, Impellizzeri FM, Sieverding M, Loibl M, von Knoch F, Mannion AF et al (2009) The 12-item Oxford Knee Score: cross-cultural adaptation into German and assessment of its psychometric properties in patients with osteoarthritis of the knee. Osteoarthr Cartil 17:49-52

29. Parsley BS, Conditt MA, Bertolusso R, Noble PC (2006) Posterior cruciate ligament substitution is not essential for excellent postoperative outcomes in total knee arthroplasty. J Arthroplasty 21:127-131

30. Tang YH, Wong WK, Wong HL (2014) Patellar clunk syndrome in fixed-bearing posterior-stabilised versus cruciate-substituting prostheses. J Orthop Surg 22:80-83

31. Vertullo CJ, Lewis PL, Lorimer M, Graves SE (2017) The effect on long-term survivorship of surgeon preference for posteriorstabilized or minimally stabilized total knee replacement: an analysis of 63,416 prostheses from the Australian Orthopaedic Association National Joint Replacement Registry. J Bone Jt Surg 99:1129-1139

32. Wajsfisz A, Biau D, Boisrenoult P, Beaufils P (2010) Comparative study of intraoperative knee flexion with three different TKR designs. Orthop Traumatol Surg Res 96:242-248

33. Yacovelli S, Grau LC, Hozack WJ, Courtney PM (2021) Functional outcomes are comparable between posterior stabilized and cruciate-substituting total knee arthroplasty designs at short-term follow-up. J Arthroplasty 36:986-990

Publisher's Note Springer Nature remains neutral with regard to jurisdictional claims in published maps and institutional affiliations. 